which parts of the insulin molecule are inseparable from hormonal function. It is known that the $\mathrm{N}$-terminal end of the B-chain and the C-terminal end of the A-chain are not essential for activity. In spite of interactions observed crystallographically between the $\mathrm{N}$ terminal ends of the two chains, the last three residues of the $\mathrm{B}$-chain can in fact be removed with little consequence in terms of activity. The new work shows that generation of insulin from intact B-chains and a synthetic A-chain, lacking the last four residues at the $\mathrm{N}$-terminus, is quite inactive. Evidently therefore this part of the molecule is important for biological activity.

\section{LASERS}

\section{Tuning Revolution}

from a Correspondent

RESEARCH into tunable lasers has made striking progress in the past two years, and the current development of such coherent sources became clear during a conference organized by the Institute of Physics and held at Heriot-Watt University, Edinburgh, between September 6 and 8 . The conference revealed that tunable coherent sources could be found for almost all parts of the electromagnetic spectrum from $0.25 \mu \mathrm{m}$ to $300 \mu \mathrm{m}$.

The conference took the form of a series of invited and contributed papers covering techniques applicable in different spectral regions, and included dye lasers, non-linear optical techniques such as optical parametric oscillators and difference frequency mixing, semiconductor diode lasers and the spinflip Raman laser. Dr B. B. Snavely (Eastman Kodak, Rochester, New York) began the conference with an account of progress in the development of continuous wave dye lasers. These can now provide tunable radiation of about $1 \mathrm{~W}$ between $0.55 \mu \mathrm{m}$ and 0.7 $\mu \mathrm{m}$, and he foresaw extensions to the near ultraviolet and infrared regions up to $1 \mu \mathrm{m}$. Pulsed dye lasers, discussed by Dr E. G. Arthurs (Queen's University, Belfast), provide considerably higher powers, in the megawatt range, in pulses of duration between $10^{-12} \mathrm{~s}$ and $10^{-6} \mathrm{~s}$. The range covered with one dye is typically 50 to $100 \mathrm{~nm}$, but by utilizing different dyes and pumping lasers a total range from $0.25 \mu \mathrm{m}$ to $1.2 \mu \mathrm{m}$ may be achieved. The dye laser also seems likely to be capable of obtaining increasingly shorter pulse durations, because of the inherently broad-band nature of the laser transition which could provide, in principle, pulse durations of less than $10^{-13} \mathrm{~s}$, although the results currently achieved are around a few picoseconds $\left(10^{-12} \mathrm{~s}\right)$.

The region of the spectrum which generated most interest and discussion was that extending from $1 \mu \mathrm{m}$ to 25 $\mu \mathrm{m}$. It is in this region that most molecules exhibit their vibration-rotation "fingerprint" - indeed, usually three times over-and therefore the impact of tunable coherent sources, with their million-fold improvement over conventional sources and spectrometers in spectral brightness (power per unit bandwidth) and resolution, becomes most significant. Already, the entire range is covered at low powers (milliwatts continuous wave) with semiconductor diode lasers, although, as was made clear by Dr E. D. Hinkley (Massachusetts Institute of Technology), a large number of separate diodes are required to fill in the whole range. Parametric oscillators and new nonlinear materials were discussed by $\mathrm{Dr}$ D. C. Hanna and Dr R. C. Smith (Southampton University) who showed that high powers, of the order of a few kilowatts, with pulsed operation were available over a considerable tuning range, 1 to $4 \mu \mathrm{m}$ and 8 to $12 \mu \mathrm{m}$. Recent experiments at Southampton have indicated that, by combining parametric oscillation with difference frequency mixing, continuous tuning from $2 \mu \mathrm{m}$ to $20 \mu \mathrm{m}$ should be possible.

By far the highest powers and narrowest linewidths currently available in this region are provided by the output from the spin-flip Raman (SFR) laser. In his paper Professor S. D. Smith (Heriot-Watt University) emphasized the versatility of this device, which at present may be tuned from 5 to 6 $\mu \mathrm{m}$ and from 9 to $14 \mu \mathrm{m}$. Over the first of these ranges, continuous wave operation with linewidths down to 1 $\mathrm{kHz}$ (less than $10^{-7} \mathrm{~cm}^{-1}$ ) have been measured, and in the latter range peak pulsed powers of around $1 \mathrm{~kW}$ have already been achieved, together with a linewidth of a few hundredths of a wavenumber. Extensions to cover the entire far infrared are current possibilities.

In an invited paper covering applications of the spin-flip Raman (SFR) laser to gaseous pollution monitoring, Dr L. B. Kreuzer (Bell Telephone
Laboratories, New Jersey) discussed his use of the SFR laser with a spectrophone to detect trace quantities of gases (such as $\mathrm{NO}, \mathrm{C}_{2} \mathrm{H}_{4}, \mathrm{SO}_{2}$, and so on) down to concentrations smaller than one part in $10^{9}$. Outstanding improvements in sensitivity and selectivity were reported. The impact of the narrow linewidths on quantitative estimation of one constituent of a mixture without separation was most impressive. As most gases possess characteristic absorption spectra even in a restricted region of, say, 5 to $6 \mu \mathrm{m}$, development work on the SFR laser could well result in a superior alternative to the conventional mass spectrometer. Dr Kreuzer discussed the use of the laser in combination with a gas chromatograph for high resolution, short time measurements of the resulting fragments.

The conference closed with a panel discussion led by the conference secretary, Dr M. J. Colles (Heriot-Watt University), which summarized the three days of proceedings and presented conference participants with a unified picture of the techniques now available throughout the spectrum. Having established the present state of the art, the panel was invited to speculate on what might happen in the next two years. The opinions indicated that tunable coherent sources would provide complete coverage from $0.2 \mu \mathrm{m}$ to $1,000 \mu \mathrm{m}$ and that a revolution in spectroscopic methods seemed inevitable.

\section{SOLID STATE \\ New Ways with Electrons}

from a Correspondent

A NEW method of mapping the Fermi velocities of electrons in a metal, involving the measurements of microwave power, has been devised by Falk et al. (Phys. Rev. B., 6, 377; 1972).

Most experiments designed to study the behaviour of electrons in metals yield information in the form of some average over a large number of electrons. Although the electrons that par-

\title{
Understanding Aromatic Cage Molecules
}

THE bonding in the planar unsaturated sulphur-nitrogen species $\left(\mathrm{S}_{2} \mathrm{~N}_{2}, \mathrm{~S}_{4} \mathrm{~N}_{3}{ }^{+}\right.$ and $\mathrm{S}_{5} \mathrm{~N}_{5}{ }^{+}$) can be rationalized in terms of the simple Hückel theory, for there are $4 n+2 \pi$-electrons, just as in aromatic hydrocarbons. In the related unsaturated cage molecules, typified by $\mathrm{S}_{4} \mathrm{~N}_{4}$ and $\mathrm{S}_{8}{ }^{2+}$, the nature of the delocalized bonding is, however, much less clear, and the problem is complicated by the presence of interatomic distances intermediate between normal single bond lengths and van der Waals separations.

In a communication in next Monday's
Nature Physical Science, Banister shows that the observed geometry of these molecules can be interpreted in terms of a flexible $\sigma$-bonded framework in which sulphur atoms lie at some or all of the corners of an approximately regular polyhedron. After subtracting $\sigma$-bonding electrons and electron lone pairs, it is found that each polyhedron gives the same number of higher energy bonding orbitals as electron pairs remaining for delocalized bonding. This approach may well improve the understanding of these apparently illogical aromatic cage molecules. 